\title{
Cross LAYER Protocols For Multimedia TRANSMISSION IN WIRELESS NETWORKS
}

\author{
Santhosha Rao ${ }^{1}$, Kumara Shama ${ }^{2}$ \\ ${ }^{1}$ Department of I\&CT, MIT, Manipal University, Manipal, India \\ msanthosharao@gmail.com \\ ${ }^{2}$ Department of E\&C Engg., MIT, Manipal University, Manipal, India \\ shama.kumar@manipal.edu
}

\begin{abstract}
Wireless networks initially inherited the traditional layered architecture from wired networks. Nevertheless, as third and fourth generation wireless communications and networking begin to proliferate in the area of communication networks, the suitability of the layered architecture is coming under close scrutiny from the research community. It is repeatedly argued that although layered architectures have served well for wired networks, they might not be suitable for wireless networks [1]. One of the key challenges for next-generation broadband wireless networks is to devise end-to-end protocol solutions across wired and wireless networks to accommodate large densities of highly mobile users demanding services and applications with a wide range of Quality of Service $(Q o S)$ requirements. Hence, in order to meet the challenging demands on future wireless networks, it may be required to adopt new approaches in which protocols can be designed by violating the reference layered architecture allowing direct communication between protocols in nonadjacent layers Such violations of a layered architecture have been termed as cross-layer design(CLD).
\end{abstract}

\section{KEYWORDS}

Cross Layer Design, EDCA Networks, FEC

\section{INTRODUCTION}

Over the last few years ample new protocols have been developed for multimedia applications in the whole OSI layer's scale. In addition wireless communications and networking fast occupy centre stage in research and development activity in the area of communication networks. In order to support the wireless user in a better way, the cross-layer design paradigm has been proposed. Multimedia data transmission experience a number of constraints that result to low Quality of Service (QoS) that is offered to the end user. These constraints have mainly to do with the nature of multimedia applications, which are characterized by three main properties: the demand for high data transmission rate (bandwidth-consuming applications), the sensitiveness to packet delays (latency and jitter) and the tolerance to packet losses (packet-loss tolerant applications), when compared to other kind of applications.

One first striking difference between wired and wireless networks is the cause of packet losses. Packet losses in wired networks occur mainly due to congestion in the path between the sender and the receiver, while in wireless networks packet losses occur mainly due to corrupted packets as a result of the low Signal to Noise Ratio (SNR), the multi-path signal fading and the interference from neighboring transmissions. A second difference between wired and wireless networks is the "mobility factor". Mobility in wireless networks introduces a number of additional barriers in multimedia data transmission. The above properties introduce new design challenges to the networking world as it is in fact difficult to combine guaranteed high bit rates and an acceptable packet loss ratio with low latency and jitter. All the above factors have led both the research community and the industry to develop and propose a number of new

DOI : $10.5121 /$ ijcses.2012.3302 
protocols and optimization techniques targeting at mitigating delay and packet loss ratio during the transmission of multimedia data. Most of these efforts are based on the classic layered approach in which the various layers try to optimize its performance by adapting its behaviour to constantly varying network parameters and provide its best services to upper layers.

Under this layered approach, communication occurs between two adjacent layers without taking, however, into consideration the specific characteristics of multimedia applications. Although this layered approach has been the fundamental factor for the growth of the wired networks it seems to pose serious constraints when attempting to adapt protocol's behaviour to multimedia application's characteristics and to wireless network conditions. For example, packet losses in wireless networks occur mainly due to corrupted packets and any typical congestion control mechanism cannot function properly when it is not aware of the cause of packet losses. In this simple example, it is obvious that someone should employ different mechanisms in which all layers share knowledge with each other about the specific multimedia application characteristics and the instant network conditions.

Cross-layer design (CLD) [2] is a new paradigm in network architecture design that takes into account the dependencies and interactions among layers, and supports optimization across layer boundaries $[3,4]$.

\section{Understanding The Cross Layer Design}

\subsection{A definition of Cross-layer Design}

A layered architecture, like the seven-layer open systems interconnect (OSI) model divides the overall networking task into layers and defines a hierarchy of services to be provided by the individual layers. The services at the layers are realized by designing protocols for the different layers. The architecture forbids direct communication between nonadjacent layers. Communication between adjacent layers is limited to procedure calls and responses. In the framework of a reference layered architecture, the designer has two choices at the time of protocol design. Protocols can be designed by respecting the rules of the reference architecture. In a layered architecture, this would mean designing protocols such that a higher-layer protocol only makes use of the services at the lower layers and is not concerned about the details of how the service is being provided. Alternatively, protocols can be designed by violating the reference architecture, for example, by allowing direct communication between protocols at nonadjacent layers or sharing variables between layers. Such violation of a layered architecture is cross-layer design with respect to the reference architecture.

\subsection{General motivation for Cross-layer Design}

The presence of wireless links in the network motivate designers to violate the layered architectures, owing to three main reasons: the unique problems created by wireless links, the possibility of opportunistic communication on wireless links, and the new modalities of communication offered by the wireless medium.

On the pessimistic side, wireless links create several new problems for protocol design that cannot be handled well in the framework of the layered architectures. In the classic case of a TCP, sender mistaking a packet error on a wireless link to be an indicator of network congestion is an example. On the optimistic side, wireless networks offer several avenues for opportunistic communication that cannot be exploited sufficiently in a strictly layered design. For instance, the time-varying link quality allows opportunistic usage of the channel whereby the transmission parameters can be dynamically adjusted according to the variations the channel quality, just to name one example. 


\subsection{Network elements involved in the adaptation process}

In multimedia transmission three entities can be distinguished that take part in the information exchange procedure: the sender, the core network elements (links, routers) and the receiver. The most challenging and may be the most beneficial approach would be the participation of all three elements in the adaptation mechanism especially when the multimedia data are transmitted among various network domains. However, even in the same network domain someone has to decide whether or not both the sender and the receiver should participate in the adaptation process. Someone should also consider that the complexity increases when interdomain adaptation and policies are to be implemented. Clearly, there are pros and cons in either approach. With both the sender and the receiver participating in the adaptation process better results are expected as this sender/receiver pair acts as an organized "team" by sharing information related to current network conditions and adapt their behaviors to these conditions. Logically, the total result would provide the highest QoS for given network conditions. On the other hand, by confining the adaptation process only in the sender or the receiver the level of independence is increased between the entities involved in the multimedia transmission. Therefore, the cross-layer adaptation scheme that is related to the participation of the entities involved in the multimedia transmission can fall into the next four categories:

- Sender based: The sender performs the cross-layer adaptation. This approach has the advantage of easy deployment due to the fact that it does not require any support from the network or the receivers. On the other hand this approach has limited capabilities.

- Receiver based: The receiver performs the cross-layer adaptation. This approach also has the advantage of easy deployment due to the fact that it does not require any support from the network or the sender. Again this approach has limited capabilities.

- Network supported: The network elements are involved in the cross-layer adaptation. In a heterogeneous environment such as the Internet, agreements have to be set up amongst the various network domains to ensure any cross-layered implementation across the path between the sender and the receiver.

- Hybrid: A combination of two or more of the above approaches. This approach is the most complicated to be implemented but has the potential to provide better performance.

\subsection{Layers involved in the cross-layer adaptation (Interlayer optimization)}

Most of the available bibliography focuses on a joint PHY and MAC layers adaptation. It has been explained in [5] that PHY and MAC layers are very important especially in wireless networks and must be taken into account during cross-layer adaptation and optimization. Moreover, the APP layer has been used in several cross-layer adaptation schemes. While the above mentioned layers (PHY, MAC and APP) have been extensively researched in cross-layer adaptation schemes. There has been little work done in the whole protocol stack.

\subsection{Parameters involved in the Cross-layer Adaptation}

Each layer offers a number of different parameters through which adaptation can be achieved. The optimization of each layer parameters includes the selection of the applicable parameters which could lead to better results. At this point, we should mention that the adaptation of a parameter in one layer may and most likely, will influence the parameters in other layers. Therefore, the adaptation of the parameters in each layer should be done by taking into account of the above mentioned assumption. The Table. 1 shows the various parameters that can be involved in cross layer adaptation. 
International Journal of Computer Science \& Engineering Survey (IJCSES) Vol.3, No.3, June 2012

Table 1: Parameters for cross layer adaptation in wireless networks

\begin{tabular}{|l|l|}
\hline Layer & Parameters \\
\hline Physical & Signal Modulation \\
\hline MAC & ARQ, FEC, QoS (IEEE 802.11e) \\
\hline Network & QoS (DiffServ, IntServ) \\
\hline Transport/Session & Adaptive Transmission Rates \\
\hline Application & Encoding Parameters \\
\hline
\end{tabular}

\subsection{Adaptation strategy}

Another important issue is how the adaptation strategy could be realized. There are various approaches in this field as following:

- Integrated approach: This approach is the most challenging because the adaptation strategy is decided jointly by all the layers.

- MAC-centric approach: In this approach, the APP layer passes its traffic information and requirements to the MAC layer that decides which APP layer packets/flows should be transmitted and at what QoS level.

- Top-down approach: In this approach, the APP layer informs the lower layers for the importance of each data packet and the lower layers treat each data set with a different way, based on QoS criteria. The higher layer protocols optimize the parameters and the strategies of the next lower layer. The top-down approach typically passes priority labels to the lower layers, which perform, for instance, class-based queuing and priority-based transmission.

- Bottom-up approach: In this approach, the lower layers (PHY and MAC) provide the upper layers with optimal services by reducing the transmission errors. The bottom-up approach typically exploits information about the current channel situation to adapt the transmission policy of the application.

The above cross-layer approaches exhibit different advantages and drawbacks for wireless multimedia transmission, and the best solution depends on the application requirements, used protocols, algorithms at the various layers, complexity and limitations.

\subsection{Device constraints}

The decision on the above mentioned design issues must be done under the following constraints:

- Device constraints: Mobile devices have many limitations when compared to desktop systems. These include display limitations, CPU resources and power consumption.

- Network constraints: Network constraints include available bandwidth, delay, RTT and QoS support.

- Application constraints: Application constraints include maximum and acceptable delay, maximum and acceptable delay jitter (especially for interactive applications), maximum and acceptable packet loss ratio and finally bandwidth constraints. 
In conclusion, the main objective of the optimization process is the optimal selection of the above described parameters in order to provide the best multimedia experience to the end user by taking into account the above described constraints.

\subsection{A Typical Cross-layer Architecture}

A typical Cross -layer Architecture [2] is depicted in Figure.1. The following steps are followed in Cross-layer optimization:

Layer abstraction: To compute an abstraction of layer-specific parameters. The number of parameters used by the Cross -Layer Optimizer is significantly reduced by this abstraction operation.

Optimization: To find the values of layer parameters that optimize a specific objective function.

Layer reconfiguration: Used to distribute the optimal values of the abstracted parameters to the corresponding layers. It is the responsibility of the individual layers to translate the selected abstracted parameters back into layer-specific parameters and actual modes of operation. These steps are repeated at a rate that depends on how fast the application requirements and transmission capabilities of the physical medium vary. Identifying the parameters that describe the capabilities of a layer is an important step. From a system perspective, there are various kinds of parameters involved, which can be classified as follows:

Directly tunable (DT) parameters: These can be set directly as a result of the Cross Layer Optimization. Examples: time slot assignment in a time-division multiple access (TDMA) system or carrier assignment in an OFDM system.

Indirectly tunable (IT) parameters: These cannot be set directly as a result of the Cross Layer Optimization, but may change as a result of the setting of DT parameters. Example: bit error rate that depends on the type of coding and modulation scheme adopted.

Descriptive (D) parameters: These can be read by the Cross -Layer Optimization, but cannot be tuned. Examples: frame rate or picture size in streaming video applications that are set at encoding time, channel quality estimates obtained from channel estimation.

Abstracted (A) parameters: These are abstractions of descriptive, DT, and IT parameters used in the Cross Layer Optimizer. Example: net transmission rate and transition probabilities of a two-state packet erasure model (Gilbert-Elliot model).

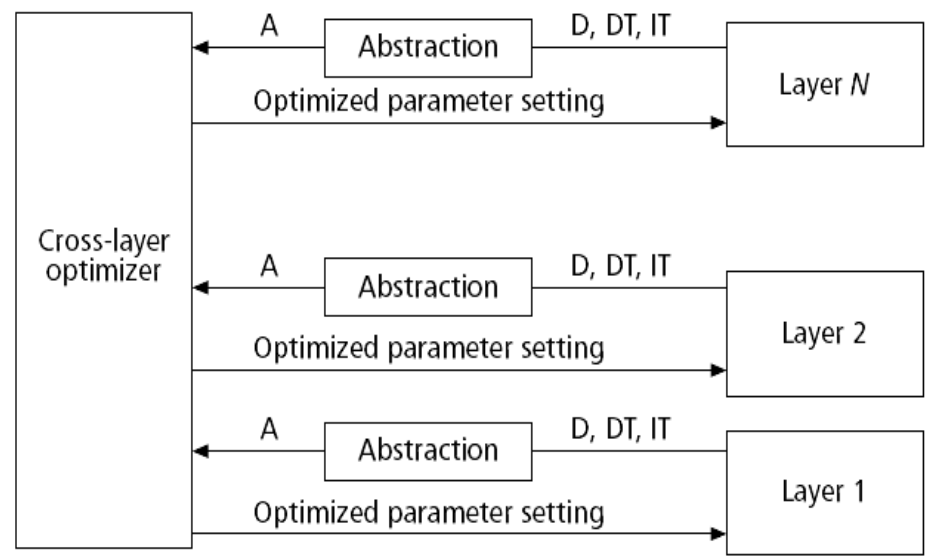

Figure. 1: A cross layer Architecture [2] 


\subsection{A snapshot of Cross-Layer Design proposals}

Layered architecture can be violated in the following basic ways:

i) Creation of new interfaces

ii) Merging of adjacent layers

iii) Design coupling without new interfaces

iv) Vertical calibration across layers

Most cross-layer design proposals in the literature fit into one of these basic categories. We shall now discuss the aforementioned four categories in more detail and point out some relevant examples. The reference layered architecture we assume is a five-layer model, with the application layer, transport layer, network layer, link layer (comprising the Data Link Control [DLC] and Medium Access Control [MAC] sublayers) and physical layer; we assume that all the layers perform their generally understood functionalities.

\subsubsection{Creation of New Interfaces}

Several cross-layer designs require creation of new interfaces between the layers. The new interfaces are used for information sharing between the layers at runtime. The architecture violation here is obviously the creation of a new interface not available in the layered architecture. Further, this category can be divided into following three subcategories depending on the direction of information flow along the new interfaces:

Upward Information Flow - A higher-layer protocol that requires some information from the lower layer(s) at runtime results in the creation of a new interface from the lower layer(s) to the higher layer. For instance, if the end-to-end TCP path contains a wireless link, errors on the wireless link can trick the TCP sender into making erroneous inferences about the congestion in the network, and as a result the performance deteriorates. Creating interfaces from the lower layers to the transport layer to enable explicit notifications alleviates such situations. For example, the explicit congestion notification (ECN) from the router to the transport layer at the TCP sender can explicitly tell the TCP sender if there is congestion in the network to enable it to differentiate between errors on the wireless link and network congestion [7]. Examples of similar upward information flow are also seen in the literature at the MAC layer in form of channel-adaptive modulation or link adaptation schemes [8]. The idea is to adapt the parameters of the transmission (e.g., power, modulation, code rate) in response to the channel condition, which is made known to the MAC layer by an interface from the physical layer.

Downward Information Flow - Some cross-layer design proposals rely on setting parameters on the lower layer of the stack at runtime using a direct interface from some higher layer. As an example, applications can inform the link layer about their delay requirements, and the link layer can then treat packets from delay-sensitive applications with priority [9].A good way to look at the upward and downward information flow is to treat them as notifications and hints, respectively, as proposed in [10]. Upward information flow serves the purpose of notifying the higher layers about the underlying network conditions; downward information flow is meant to provide hints to the lower layers about how the application data should be processed.

Back and Forth Information Flow - Two layers, performing different tasks, can collaborate with each other at runtime. Often, this manifests in an iterative loop between the two layers, with information flowing back and forth between them. Clearly, the architecture violation here is the two complimentary new interfaces. As an example, we refer to the Network-Assisted Diversity Multiple Access (NDMA) proposal [11], whereby the physical PHY and MAC layers collaborate in collision resolution in the uplink of a wireless LAN system. Basically, with improvements in the signal processing at the PHY, it becomes capable of recovering packets from collisions. Thus, upon detecting a collision the base station first estimates the number of 
users that have collided, and then requests a suitable number of retransmissions from the set of colliding users. Then PHY signal processing lets the base station separate the signals from all the colliding users.

\subsubsection{Merging of Adjacent Layers}

Another way to do cross-layer design is to design two or more adjacent layers together such that the service provided by the new superlayer is the union of the services provided by the constituent layers. This does not require any new interfaces to be created in the stack. Architecturally speaking, the superlayer can be interfaced with the rest of the stack using the interfaces that already exist in the original architecture.

\subsubsection{Design coupling without new interfaces}

Another category of cross-layer design involves coupling two or more layers at design time without creating any extra interfaces for information sharing at runtime. While no new interfaces are created, the architectural cost here is that it may not be possible to replace one layer without making corresponding changes to another layer. For instance, [12] considers the design of a MAC layer for the uplink of a wireless LAN when the PHY is capable of providing multipacket reception capability. Multipacket reception capability implies that the PHY is capable of receiving more than one packet at the same time.

\subsubsection{Vertical Calibration across the Layers}

As the name suggests, this refers to adjusting parameters that span across layers. The motivation is easy to understand. Basically, the performance seen at the level of the application is a function of the parameters at all the layers below it. Hence, it is conceivable that joint tuning can help to achieve better performance than individual settings of parameters as would happen had the protocols been designed independently can achieve. Vertical calibration can be done in a static manner, which means setting parameters across the layers at design time with the optimization of some metric in mind. It can also be done dynamically at runtime, which emulates a flexible protocol stack that responds to variations in the channel, traffic, and overall network conditions.

\subsection{Proposals for implementing Cross-layer interactions}

Basically there are three proposals for the implementation of Cross-layer interactions:

i) Direct communication between layers

ii) A shared database across the layers

iii) Completely new abstractions

\subsubsection{Direct communication between layers}

A straightforward way to allow runtime information sharing between layers is to allow them to communicate with each other, as depicted schematically in Figure.2. Note that this is applicable when there has to be runtime information sharing between layers (e.g., in cross-layer designs that rely on new interfaces or in dynamic vertical calibrations). Practically speaking, direct communication between the layers means making the variables at one layer visible to the other layers at runtime.

\subsubsection{A shared database across the layers}

This proposes a common database that can be accessed by all layers, as illustrated in Figure.2 (e.g., [13]). The common database is like a new layer, providing the service of storage/retrieval of information to all the layers. The shared database approach is particularly well suited to vertical calibrations across layers. An optimization program can interface with the different 
layers at once through the shared database. Similarly, new interfaces between the layers can also be realized through the shared database.

\subsubsection{Completely new Abstractions}

It presents a new way to organize the protocols: in heaps, not in stacks as done by layering. Such novel organizations of protocols are appealing as they allow rich interactions between the building blocks of the protocols. Hence, potentially they offer great flexibility, both during design as well as at runtime [14]. However, they change the very way protocols have been organized, and hence may require completely new system-level implementations.

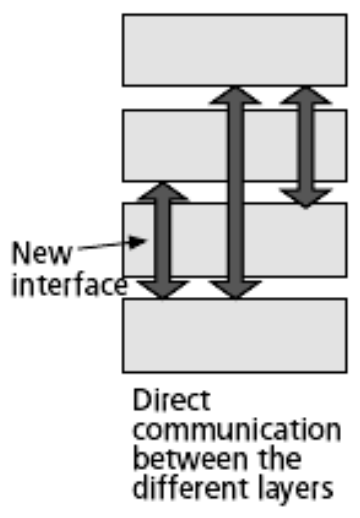

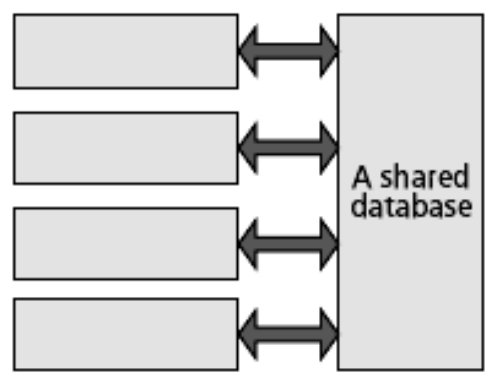

A shared database

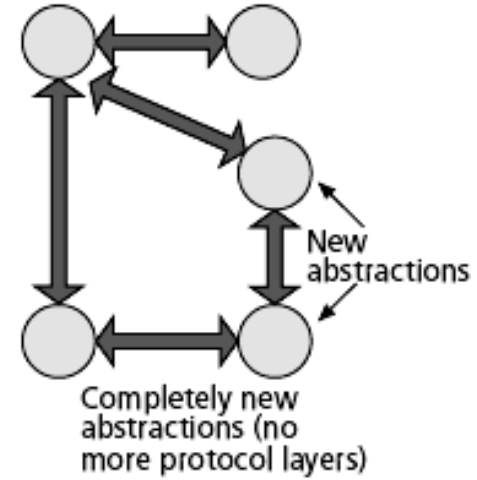

Figure. 2 Proposals for implementing cross layer interactions

\section{SNapshot Of Cross-Layer Solutions For Wireless Video COMMUNICATIONS}

The previous section has presented some cross-layer architectures that improve the performance of the transmission adapting the parameter setting of each layer through an inter-layer message exchange. In the following paragraphs we will focus on video transmission, and will present some of the cross-layer schemes that have been designed to allow a reliable transmission over wireless networks.

\subsection{The joint source-channel coding (JSCC) approach}

The joint source-channel coding (JSCC) is a possible approach to effectively reduce the errors occurred during transmission by allocating the resources between source codes and channel codes (bit allocation problem) [15]. It is important to derive an analytic model describing the relation between media quality and source/channel parameters. The most common metric to evaluate media quality is the expected end-to-end distortion $\mathrm{D}_{\mathrm{T}}$, where $\mathrm{D}_{\mathrm{T}}$ consists of source distortion $\mathrm{D}_{\mathrm{S}}$ and channel distortion $\mathrm{D}_{\mathrm{C}}$. Source distortion is caused during the media source encoding (i.e., quantization, motion estimation, rate control). Channel distortion occurs when parts of media stream are lost due to network congestion, or incorrectly received due to wireless channel noise. Therefore, the bit allocation problem can be formulated as the optimization problem:

$\min \mathrm{D}_{\mathrm{T}}\left(\mathrm{D}_{\mathrm{S}}, \mathrm{D}_{\mathrm{C}}\right)$ s.t. $\mathrm{R}_{\mathrm{S}}+\mathrm{R}_{\mathrm{C}} \leq \mathrm{R}_{\mathrm{T}}$

Where $R_{T}$ is the total available bandwidth, and $R_{S}$ and $R_{C}$ are the rates for source coding and channel coding, respectively. JSCC schemes are thus proposed to achieve the optimal end-toend quality by adjusting the source and channel coding parameters, simultaneously. 


\subsection{Cross-layer optimization between application, data link and physical layers}

In [16], [17] and [18] a cross-layer optimization between application layer, data link layer, and physical layer to optimize the end-to-end quality of the wireless streaming video application and to use efficiently the wireless resources, is proposed. The authors include the application layer in the joint optimization because the end-to end quality observed by the users directly depends on the application and the application layer has information about the impact of each successfully decoded piece of multimedia data on the perceived quality. The architecture analysed consists of the process of parameter abstraction, a cross-layer optimizer, and the process of decision distribution. They consider a video streaming server located at the base station and multiple streaming clients located in mobile devices. The users are sharing the same air interface and network resources but they request different video contents. At the base station, an architecture as shown in Figure. 3 is proposed to provide end-to end quality-ofservice optimization.

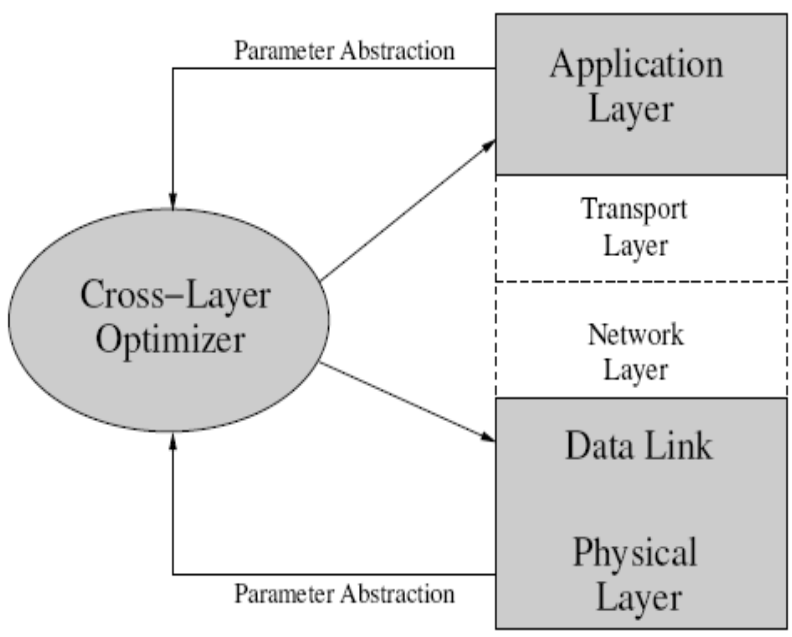

Figure. 3 Cross-Layer Architecture proposed in [18]

(i) Parameter abstraction: State information parameters are collected from the application layer and the radio link layer and they are transformed into parameters that are comprehensible for the cross-layer optimizer, so-called cross-layer parameters. In a single-user scenario, for example, the abstracted key parameters from the data link layer and physical layer could be: transmission data rate $\mathrm{d}$, transmission packet error ratio e, data packet size $\mathrm{s}$, and the channel decorrelation time t. At application level, instead, the abstracted parameters could include the source data rate, the number of frames per second, the size (in bytes), and the maximum delay of each frame. Other important information for the optimizer is the distortion-rate function and the so-called loss distortion profile, which shows the distortion $D_{i}$ that is introduced in case the $\mathrm{i}^{\text {th }}$ frame of the GOP is lost.

(ii) Cross-layer optimization: The cross-layer optimizer performs the optimization with respect to a particular objective function. From a given set of possible cross-layer parameter tuples, the tuple optimizing the objective function is selected. The choice of a particular objective function depends on the goal of the system design, and the output (or decision) of the optimizer might be different for different objective functions. In the example of streaming video, one possible objective function in a single user scenario is the MSE between the displayed and the original video sequence, that is, the sum of loss distortion $\mathrm{MSE}_{\mathrm{L}}$ and source distortion $\mathrm{MSE}_{\mathrm{S}}$ : 
$\mathrm{MSE}=\mathrm{MSE}_{\mathrm{L}}+\mathrm{MSE}_{\mathrm{S}}$

Where $\mathrm{MSE}_{\mathrm{L}}$ can be computed from the distortion profile by

$$
M S E_{L}-\sum_{i=1}^{N} D_{i} P_{i}
$$

where $\mathrm{N}$ is the number of frames in one GOP, $\mathrm{P}_{\mathrm{i}}$ is the probability that the $\mathrm{i}^{\text {th }}$ frame is the first frame lost during transmission of this GOP and $\mathrm{D}_{\mathrm{i}}$ is the mean square error that is introduced by this loss. The parameter $\mathrm{D}_{\mathrm{i}}$ is taken from the measured distortion profile and is usually different for each GOP. The parameter $\mathrm{P}_{\mathrm{i}}$ can be derived using the data link layer and physical layer parameters introduced before. For a multiuser situation, different extensions of the MSE are possible. For example, the objective function can be the sum of MSE of all the users. That is,

$$
\Gamma=\sum_{k=1}^{K} M S E_{k}
$$

where $\mathrm{MSE}_{\mathrm{k}}$ is the MSE of the user $\mathrm{k}$.

(iii) Decision: After the decision on a particular cross-layer parameter tuple is made, the optimizer distributes the decision information back to the corresponding layers.

\subsection{MAC centric Approach}

A MAC centric cross layer approach for the MPEG-4 video transmission in IEEE 802.11e network is proposed in [19]. To support the varying Quality-of-Service (QoS) requirements of emerging applications, a new standard IEEE 802.11e has been specified. The 802.11e standard defines four access categories (ACs) that have different transmission priorities. The transmission priority is the probability of successfully earning the chance to transmit when individual ACs are competing to access the wireless channel; the higher the transmission priority, the better is the opportunity to transmit. However, for a wireless channel, the unavoidable burst loss, excessive delays, and limited bandwidth become challenges for efficient multimedia transmission over wireless network. Consequently, several advanced mechanisms were proposed based on 802.11e to support multimedia transmissions and in particular video transmission quality. Most of the proposed mechanisms improved the performance by adjusting the operation of 802.11e MAC. However, the mechanisms did not exploit the significance of a specific traffic type (such as video) into consideration, thereby limiting the performance improvements that can be obtained.

For video traffic, the significance of the encoded video data varies. The priority transmission of hierarchical coding video is expected to play an important role in supporting multimedia service in a wireless network. However, 802.11e provides QoS through traffic distribution where all video data in the same access category. As a result, the channel access mechanism and the transmission scheme do not take the significance information of video data into consideration. If the transmission mechanism exploits the characteristics of video data content by considering the video significance information generated from the application layer, the video data will have priority service and the perceived quality at the receiver side can be improved.

The MPEG-4 standard defines three types of video frames for the compressed video stream, including I (Intra-coded) frame, P (Predictive-coded) frame, and B (Bi-directionally predictivecoded) frame. The MPEG I frame is encoded independently and decoded by itself. Thus, the I frame is just a frame coded as a still image, without any relationship to any previous or successive frames. The $\mathrm{P}$ frame is encoded using prediction from the preceding I or P frame in the video sequence. Thus the $\mathrm{P}$ frame requires the information of the most recent $\mathrm{I}$ frame or $\mathrm{P}$ frame for encoding and decoding. The B frame is encoded using predictions from the preceding 
and succeeding I or P frames. According to the coding relation, in MPEG-4 video stream the most important video type is the I frame, with the $\mathrm{P}$ frame being more important than $\mathrm{B}$ frame. A GOP pattern is characterized by two parameters, $\mathrm{G}(\mathrm{N}, \mathrm{M})$ : N is the I-to-I frame distance and $\mathrm{M}$ is the I-to-P frame distance. For example, G $(9,3)$ means that the GOP includes one I frame, two $P$ frames, and six B frames. Similarly, the second I frame in the figure marks the beginning of the next GOP. The arrows indicate that the B frames and $\mathrm{P}$ frames decoded are dependent on the preceding or succeeding I or P frames. Prediction encoding of MPEG-4 is shown in Figure 4 .

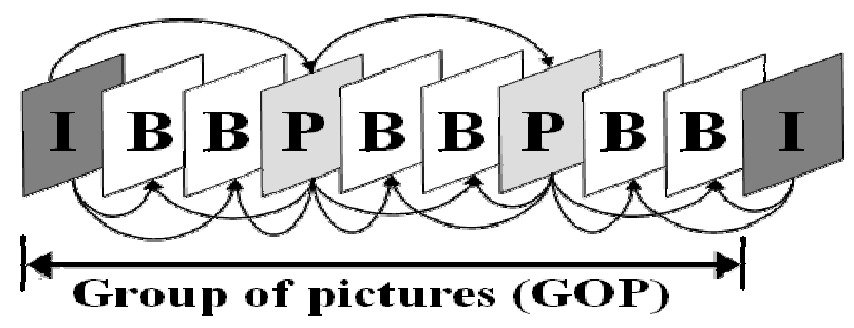

Figure. 4 : Prediction encoding of MPEG-4, GOP (N=9, M=3)

As shown in Figure. 5, the authors in [19], proposed a mapping algorithm, based on the traffic specification of IEEE 802.11e EDCA, and encoded H.264 video data is allocated into different precedence AC queues according to the video coding significance.

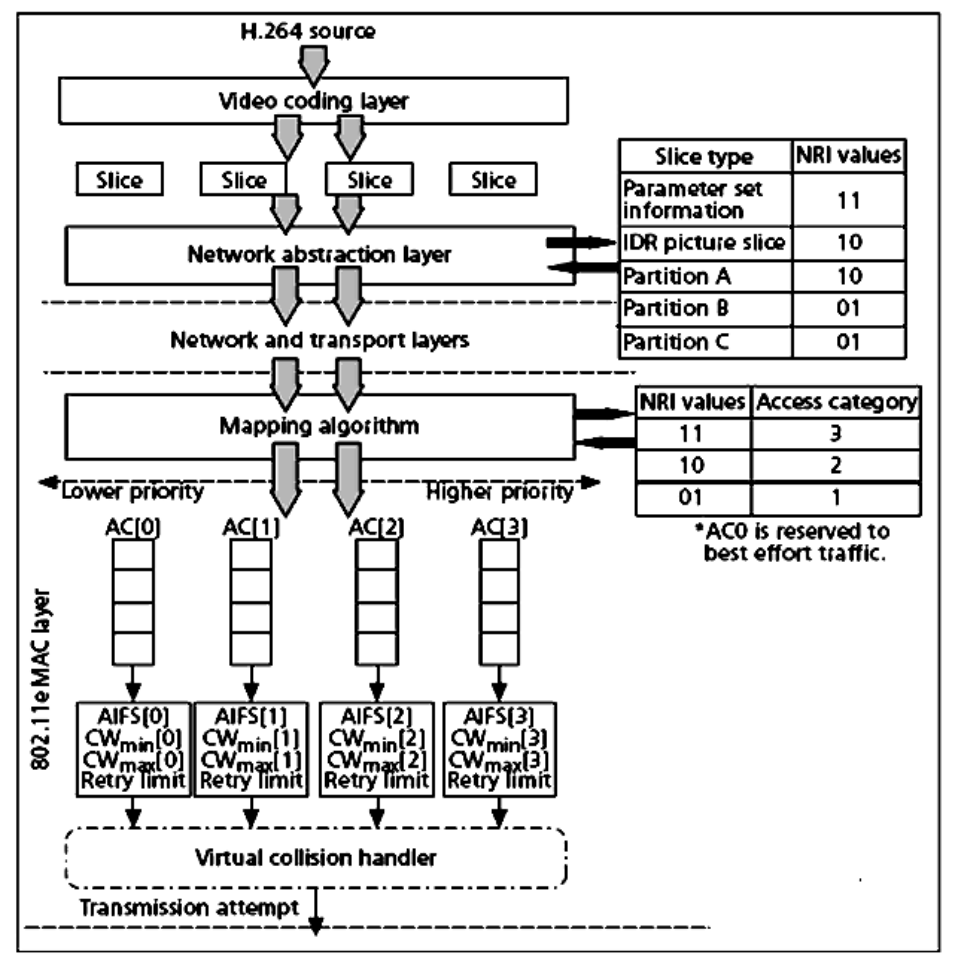

Figure. 5 A cross layer architecture for video transmission in IEEE 802.11e networks 


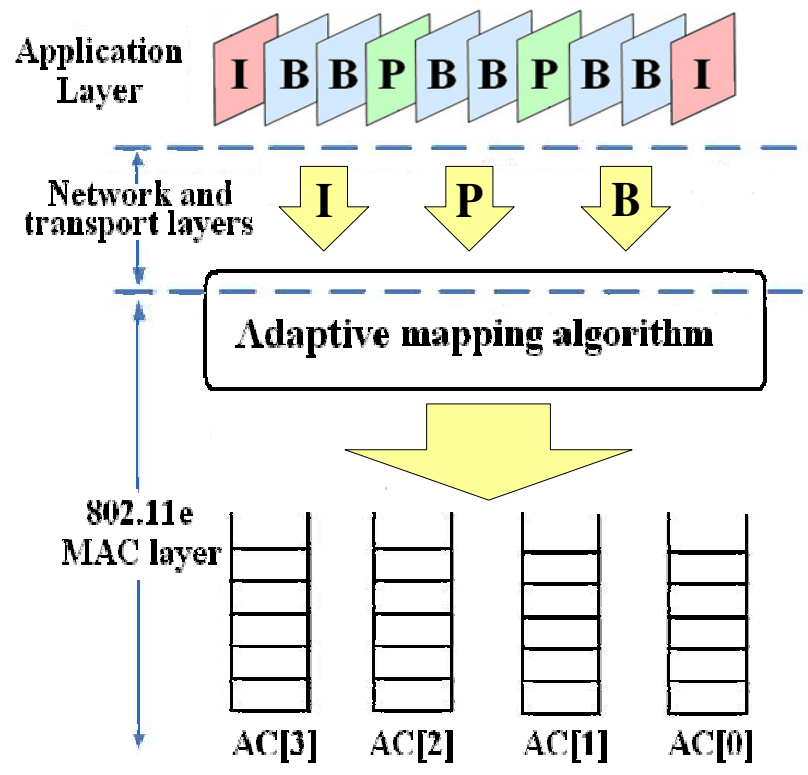

Figure.6 Architecture of adaptive cross-layer mapping scheme

However, the mapping is static and not adaptive. When the network load is light, the video data which is mapped to lower priority AC will result in unnecessary transmission delays and packet losses. Accordingly, if MPEG-4 video streams are transmitted as the traffic for the mapping algorithm proposed in [19], the I frame will always be mapped to AC[2], while the P frame will be mapped to $\mathrm{AC}[1]$ and the $\mathrm{B}$ frame will be mapped to $\mathrm{AC}[0]$. If the $\mathrm{AC}[2]$ queue is empty (which means the video traffic load is light) such a static mapping algorithm will result in unnecessary transmission delays as well as high packet loss if $\mathrm{AC}[1]$ and $\mathrm{AC}[0]$ are almost full at the same time.

In the cross-layer approach proposed in [20], MPEG-4 video packets are dynamically mapped to the appropriate $\mathrm{AC}$ based on both the significance of the video data and the network traffic load. This is depicted in Figure.6.

To guarantee the quality of delivered video the proposed mapping algorithm dynamically allocates the video to the most appropriated $\mathrm{AC}$ at the MAC layer according to both the significance of video type and the network traffic load.

\subsection{Cross layer based FEC}

Increasing numbers of Internet users connect to Internet services with wireless components, like laptop computers and PDAs, due to their convenience. Unfortunately, wireless network packet error is unavoidable and more serious than in wired networks. Unavoidable packet errors however, are usually recovered using ARQ (Automatic Repeat Request) or FEC (Forward Error Correction) techniques. In ARQ, missing packets are retransmitted during timeouts or explicit receiver requests. In FEC, the sender prevents packet losses by transmitting redundant information, allowing reconstruction of a certain amount of missing data at the receiver without the need for retransmissions. Retransmitting lost packets in large-scale multimedia video transmission is often unfeasible as retransmission incurred delay is unacceptable.

FEC mechanisms could be classified into two classes based on how to add the redundant information to the original data. One is static FEC, and the other is dynamic FEC. For static FEC mechanisms, redundant information is added to transmission data in a fixed number. The static FEC is easier to implement. However, the static FEC disadvantage is that it does not flexibly adapt to network conditions. For dynamic FEC mechanisms, redundant information is 
dynamically added based on the network condition. The most important advantage of dynamic FEC mechanism is adaptation to network variations and leads to better system performance.

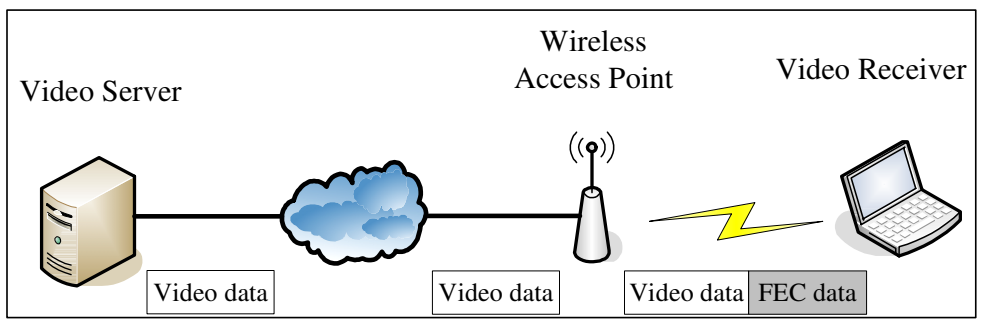

Figure. 7 Wireless AP adds redundant FEC data to video transmission data

An Adaptive Cross-layer Forward Error Correction (ACFEC) is explained in [21].This algorithm is realized in Access Point(AP) which adds FEC to video data adaptively. In the infrastructure mode, when every wired and wireless node wants to send data packets to other wireless nodes, data must first be sent to the Access Point (AP). The AP then forwards packets to the corresponding node. Video data is transmitted over the network as shown in Figure.7. The packet-level FEC encoder generates error correction packets, based on a certain number of source packets that constitute a single block. When the source packets are transmitted to the receiver through the wireless AP, the adaptive FEC controller classifies the video data packets, and groups them in blocks. Furthermore, the adaptive FEC controller monitors the transmission results of video data packets by snatching up the failure information from the MAC layer. If the transmission fails, the failure counter in the adaptive FEC controller increases by one. After transmitting one block of the video data packets, the adaptive FEC controller uses the failure counter to adjust the number of redundant FEC packets to be generated. By accurately detecting packet losses and adjusting the redundancy rates accordingly, the number of FEC packets increases or decreases to meet the need of the receiver and to overcome the packet losses. No FEC packets are generated, when the all-video data packets of one block arrive in the receiver successfully.

\section{CONCLUSION}

This paper has taken stock of the current activity in the area of cross-layer design. After suggesting a definition, we introduced the different interpretations of cross-layer design together and a taxonomy of some cross-layer design proposals. We also emphasized the initial ideas for implementing cross-layer interactions. Next, we exemplified some of the cross-layer protocols for enhancing the video quality in wireless networks.

\section{REFERENCES}

[1] V. Srivastana and M. Motani,(2005) Cross-layer design: a survey and the road ahead, IEEE Commun. Mag., vol. 43, no. 12, pp. 112-119.

[2] S. Khan, Y. Peng, E. Steinbach, M. Sgroi, and W. Kellerer,(2006) Application driven crosslayer optimization for video streaming over wireless networks, IEEE Commun. Mag., vol. 44, no. 1, pp. 122-130.

[3] S. Shakkottai, T. Rappaport, and P. Karlsson,(2003) Cross-Design for Wireless Networks, IEEE Commun. Mag., vol. 41.

[4] V. Kawadia and P. Kumar,(2005) A Cautionary Perspective on Cross Layer Design, IEEE Wireless Commun., vol. 12.

[5] Chen, J., Tiejun L. \& Zheng, H., (2004) Cross-layer design for QoS wireless communications, Proceedings of the 2004 International Symposium on Circuits and Systems, ISCAS '04. 
International Journal of Computer Science \& Engineering Survey (IJCSES) Vol.3, No.3, June 2012

[6] Radha, H., Van der Schaar, M. \& Chen, Y.,(2001) The MPEG-4 Fine- Grained Scalable video coding method for multimedia streaming over IP, IEEE Transactions on Multimedia, Vol. 3.

[7] S. Shakkottai, T. S. Rappaport, and P. C. Karlsson,(2003) Cross-Layer Design for Wireless Networks, IEEE Commun.Mag., vol. 41, no. 10.

[8] Z. Ji et al.,(2004) Exploiting Medium Access Diversity in Rate Adaptive Wireless LANs, Proc. ACM Annual Int'l. Symp. Mobile Comp. and Net., Philadelphia.

[9] G. Xylomenos and G. C. Polyzos,(2001) Quality of Service Support over Multi-service Wireless Internet Links, Comp. Net., vol. 37, no. 5.

[10] L. Larzon, U. Bodin, and O. Schelen,(2002) Hints and Notifications, Proc. IEEE Wireless Commun. and Net. Conf., Orlando.

[11] G. Dimic, N. D. Sidiropoulos, and R. Zhang,(2004) Medium Access Control — Physical CrossLayer Design, IEEE Sig. Proc., vol. 21, no. 5.

[12] L. Tong, V. Naware, and P. Venkitasubramaniam,(2004) Signal Processing in Random Access, IEEE Sig. Proc., vol. 21, no. 5.

[13] V. T. Raisinghani and S. Iyer,(2004) Cross-Layer Design Optimizations in Wireless Protocol Stacks, Comp. Commun., vol. 27.

[14] R. Braden, T. Faber, and M. Handley,(2002) From Protocol Stack to Protocol Heap — RoleBased Architecture, Proc. Hot Topics in Net., Princeton, NJ.

[15] Q. Zhang, F. Yang, and W. Zhu,(2005) Cross-layer QoS Support for Multimedia Delivery over Wireless Internet,. EURASIP Journal on Applied Signal Processing.

[16] L.-U. Choi, W. Kellerer, and E. Steinbach,(2006) On cross-layer design for streaming video delivery in multiuser wireless environments, . EURASIP Journal on Wireless Communications and Networking.

[17] L. Choi, W. Kellerer, and E. Steinbach,(2004) Cross-Layer optimization for Wireless MultiUser Video Streaming, in Proceedings of the International Conference on Image Processing (ICIP'04), Singapore.

[18] S. Khan, Y. Peng, E. Steinbach, M. Sgroi, and W. Kellerer,(2006) Application-driven crosslayer optimization for video streaming over wireless networks,. IEEE Commun. Mag., vol. 44, no. 1.

[19] Ksentini A., Naimi, M., and Gueroui A., (2006) Toward an improvement of H.264 video transmission over IEEE 802.11e through a cross-layer architecture, IEEE Communications Magazine.

[20] C-H. Lin, C-H. Ke, C-K.Shieh, N.K. Chilamkurti, S.Zeadally,(2008) A Novel Cross-Layer Architecture for MPEG-4 Video Stream over IEEE 802.11e Wireless Network, In Special Issue of International Journal of Telecommunications System.

[21] Longzhe Han1 et.al ,(2009) An Adaptive Cross-Layer FEC Mechanism for Video Transmission over 802.11 WLANs, KSII The first International Conference on Internet (ICONI).

\section{Authors}

Mr. Santhosha Rao received B.E. degree in 1998 in Electronics and Communication Engineering from Mangalore University and M.Tech degree in 2007 in Digital Electronics and Advanced Communication from Manipal University, Manipal, India. Since 1998 he has been with Manipal Institute of Technology, Manipal University, Manipal, India, where he is currently Senior Assistant Professor and research scholar in the Department of Information and Communication Technology. He has presented several research papers in National and International conferences.

Dr.Kumara Shama received B.E. degree in 1987 in Electronics and Communication Engineering and M.Tech. degree in 1992 in Digital Electronics and Advanced Communication, both from Mangalore University, India. He obtained his Ph.D Degree from Manipal University, Manipal in the year 2007 in the area of Speech Processing. Since 1987 he has been with Manipal Institute of Technology, Manipal University, Manipal, India, where he is currently a Professor and Associate Director (Placement). His research interests include Speech Processing, Digital Communication and Digital Signal Processing. He has published many Research papers in various journals and conferences. 